\title{
Measurement of Aircraft Wake Vortices Using Doppler LIDAR*
}

\author{
Takeshi OGASAWARA**,+, Takashi MISAKA**, Toshihiro OGAWA**, \\ Shigeru OBAYASHI** and Izumi YAMADA*** \\ **Institute of Fluid Science, Tohoku University, \\ 2-1-1 Katahira, Aoba-ku, Sendai 980-8577, Japan. \\ ***Electronic Navigation Research Institute, \\ 7-42-23 Jindaijihigashi-machi, Chofu, Tokyo 182-0012, Japan. \\ + E-mail: ogasawara@edge.ifs.tohoku.ac.jp
}

\begin{abstract}
In this research, the wake turbulence from actual passenger airplanes taking off from Sendai airport was measured with Electronic Navigation Research Institute's Doppler laser radar (lidar). First, the influence of the surrounding wind on the behavior of wake vortices was investigated. The wake vortices in the crosswind case disappear more quickly from the runway than those of the low surrounding wind and head wind cases. In addition, the wake vortices in the case of large crosswind move faster than those in small crosswind. Next, the correction factor was estimated by using the pseudo lidar measurements based on Computational Fluid Dynamics (CFD). The corrected data for weak surrounding wind case agreed with the existing wake vortex model.
\end{abstract}

Key words: Measurement, Aircraft, Wake Vortex, Lidar

\section{Introduction}

Wake vortices are generated from both wing tips of a flying aircraft. The flow field behind of the aircraft is perturbed by the vortices and it leads to wake turbulence. The wake turbulence generated by the leading aircraft may affect the following aircraft. Therefore, this is an important issue regarding the safety of traffic operations, especially at the airport

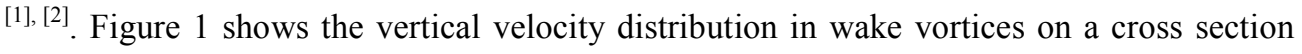
which is perpendicular to the flight path.

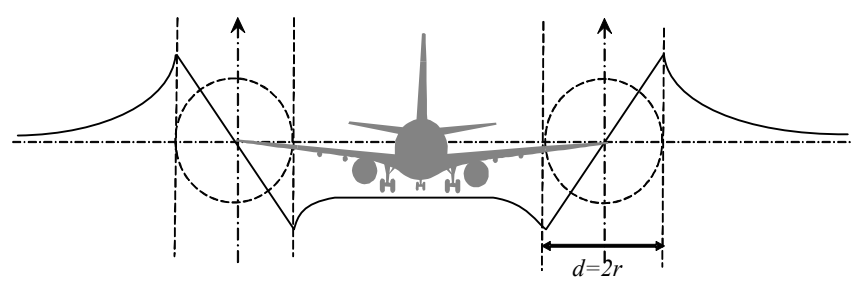

Fig. 1 Vertical velocity distribution in wake vortices

Strength of wake turbulence depends on the weight and span length of an aircraft ${ }^{[3]}$. Generally, stronger circulation of wake turbulence arises with a larger scale aircraft. If the following aircraft goes into wake turbulence, even if it is a large scale aircraft, safety and the comfort of the aircraft might be ruined. Therefore, airports set air traffic control (ATC) 
procedures for the following aircraft to avoid the danger of wake turbulence; particularly set a requirement for sufficiently safe take-off and/or landing separation minima between aircrafts as shown in Table 1 and Fig. $2^{[4]}$. However, with the growth of air traffic in recent years, the requirement for the separation minima become obstructive to efficient airport operation. Then it is indicated that the separation minima is too long in many cases ${ }^{[5]}$. If we can detect and evaluate wake turbulence quantitatively, and also consider the effect on wake turbulence caused by the surrounding atmosphere during actual traffic operations, the separation minima would be allowed to be shortened, while keeping the safety for the following aircraft.

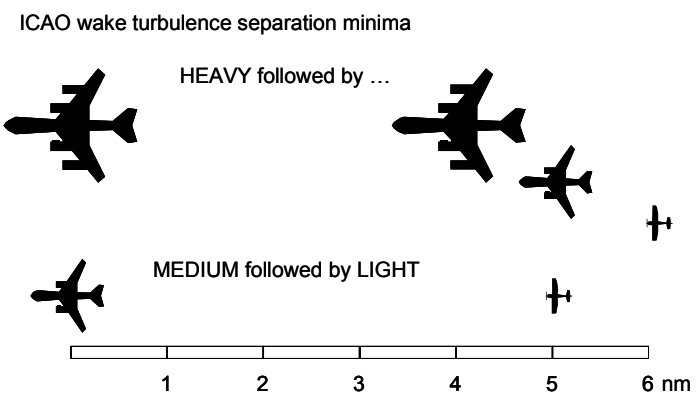

Fig. 2 Take-off and/or landing separation minima (distance) ${ }^{[4]}$

Table $1 \quad$ Take-off and/or landing separation minima (time)
\begin{tabular}{|c|c|c|}
\hline Leading Aircraft & Following Aircraft & Separation minima \\
\hline HEAVY & $\begin{array}{c}\text { HEAVY } \\
\text { MEDIUM }\end{array}$ & 2 min \\
\hline $\begin{array}{c}\text { HEAVY } \\
\text { MEDIUM }\end{array}$ & LIGHT & 3 min \\
\hline
\end{tabular}

There are several works regarding wake turbulence phenomena at airports. For example, when a weak crosswind of 1 to $5 \mathrm{kt}$ exists, wake vortices reside for a long time near the runway. In particular, when a weak wind blows from behind with a lateral angle of $45 \mathrm{deg}$, the influence of wake turbulence becomes remarkable ${ }^{[6],[7]}$. We have been measuring the wake vortices generated from actual passenger aircrafts which take off from Sendai airport using a wake turbulence detection device based on a Doppler laser radar (lidar) owned by Electronic Navigation Research Institute (ENRI) ${ }^{[8]}$. Generally, a lidar detects aerosol, which is dust and vapor in the atmosphere as a measurement object, thus it is possible to measure the wind velocity (only the component in the lidar's line-of-sight direction) even when it is a fine day ${ }^{[9]}$. The ENRI's lidar has a high resolution power in the scanning of the elevation direction. Therefore, the lidar can measure the position of a wake vortex clearly. On the other hand, the measurement data is averaged every $30 \mathrm{~m}$ the line-of-sight direction, so it results in low resolution power in this direction. This limitation is not really desirable because the core diameter of the wake vortex is generally in the range of 1 to $20 \mathrm{~m}$. Therefore, it is difficult to capture the strength and the structure of the wake vortex accurately by a simple use of the lidar measurements, although the convection of the wake vortices is captured ${ }^{[10]}$.

In this paper, the influence of the surrounding wind on the behavior of wake vortices is investigated using the ENRI's lidar for actual aircrafts at the Sendai airport. In addition, to estimate the error due to the resolution power in the line-of-sight direction, the pseudo lidar measurements are considered and applied to the artificial wake vortices that are numerically generated by the CFD simulation. Then, the discrepancy in the wake vortex parameters before and after applying the pseudo lidar measurements (straight CFD and processed CFD results) is evaluated as a correction factor. Finally, the correction factor is applied to the 
actual lidar measurements for more accurate prediction of wake turbulence.

\section{Method}

\section{A. Lidar measurements}

Figure 3 shows the external of the lidar system (Mitsubishi Electric Co. Ltd) owned by the Iwanuma branch of ENRI ${ }^{[11]}$. A specification of the lidar is shown in Table 2. The lidar has as features, a quick response for real-time measurements, a high resolution power in the elevation angle direction to capture the position of a wake vortex, and eye safe laser that can be applied against operating aircrafts.

The lidar is placed adjacent to the runway in the Sendai airport as shown in Fig. 4 to measure the wake vortices of the aircraft that is taking off. The laser light scans in the vertical direction. The scanning speed was set to $1.0 \mathrm{deg} / \mathrm{s}$, the scanning range in the direction of elevation was set to -2 to $+15 \mathrm{deg}$ and the time required for the scanning is about $20 \mathrm{~s}$. A distance from the lidar to the runway center line is $579 \mathrm{~m}$, and the flight altitude of the aircraft on the scanning plane is about $170 \mathrm{~m}$. The scanning plane is not perpendicular to the runway. In this study, the measured velocity components are simply projected onto the perpendicular runway.

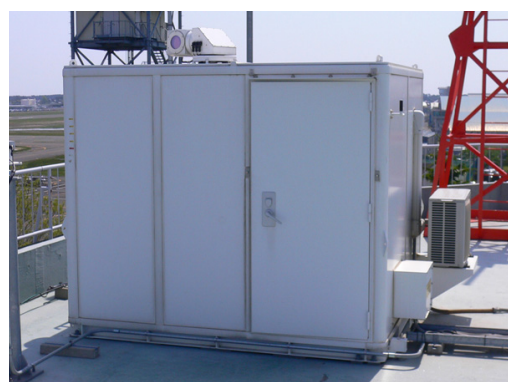

Fig. 3 Externals of the lidar

Table 2 Specification of the lidar

\begin{tabular}{lr}
\hline \hline \multicolumn{1}{c}{ Lidar system parameters } & \multicolumn{1}{c}{ Value } \\
\hline Wave length & $1.54[\mu \mathrm{m}]$ \\
Output (Average) & $2[\mathrm{~W}]$ \\
Puise width & $0.2[\mu \mathrm{s}]$ \\
Beam diameter & $100[\mathrm{~mm}]$ \\
Resolution (line-of-sight direction) & $29.9[\mathrm{~m}]$ \\
Range of velocity of the wind measurement & $\pm 30[\mathrm{~m} / \mathrm{s}]$ \\
Range of scanning (horizontal) & $0 \sim 360[\mathrm{deg}]$ \\
Range of scanning (vertical) & $-5 \sim 185[\mathrm{deg}]$ \\
Angle resolution & $0.05[\mathrm{deg}]$ \\
\hline \hline
\end{tabular}

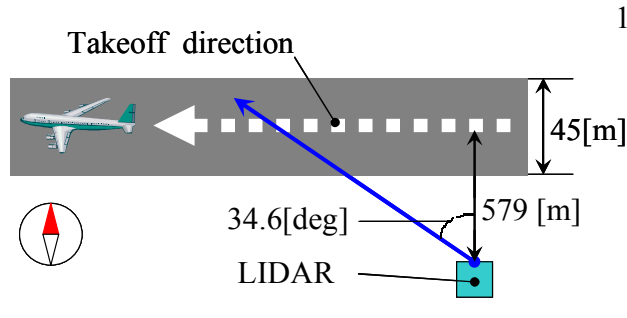

(a) Top view

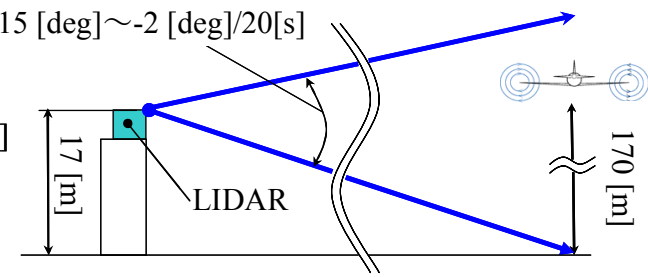

(b) Side view

Fig. 4 Setting of lidar measurements 
Figure 5 shows an example of the lidar measurement results for Boeing 767-300. This is a contour of the wind velocity component in the lidar's line-of-sight direction just after the aircraft passes through the measurement plane. In this figure, a vertical axis shows the altitude and a horizontal axis shows a horizontal distance from the lidar. A pair of wake vortices appears as four peaks in the center of this figure. The upper and lower velocity peaks of the left correspond to a clockwise vortex generated by the left wing, and those of the right correspond to a counterclockwise vortex generated by the right wing. The wake vortices seem to be enlarged horizontally and become asymmetric size, because the resolution power of the lidar in the line-of-sight direction is low, effect of wind velocity of atmosphere and scanning technique.

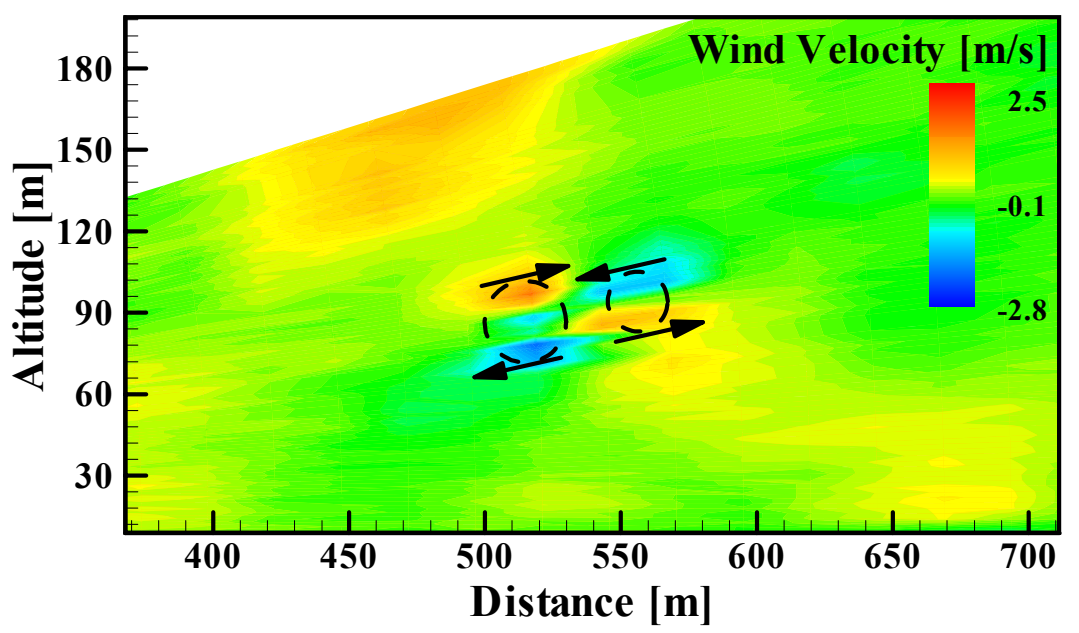

Fig. 5 Example of lidar measurements (arrows show velocity vectors of wake vortices and broken-lines show presumed wake vortices)

\section{B. Wake vortex parameters}

Wake vortex parameters are obtained from the lidar measurements as follows; the center of wake vortex is a center between the measured maximum and minimum wind velocity points. The core radius is a half of the distance between the maximum and minimum wind

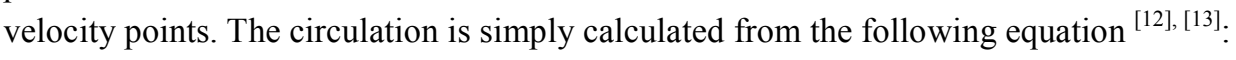

$$
\Gamma(r)=2 \pi r V(r)
$$

where $\Gamma(r)$ is the circulation, $V(r)$ is the velocity induced by the wake vortex at radius $r$. This equation is often used for the data from the measurement of line-of-sight direction. Furthermore, $V(r)$ is obtained from Eq. (2) to eliminate the surrounding wind velocity from the measured velocity:

$$
V(r)=\frac{V(r)_{+}-V(r)_{-}}{2},
$$

where $V(r)_{+}$and $V(r)_{\text {- }}$ are the measured velocity of tailwind and headwind to the lidar, and $V(r)$ is the velocity of the wake vortex. Then, the peak velocity of wake vortex is obtained from Eq. (2) with the maximum and minimum velocity at the core radius. 


\section{Pseudo lidar measurements}

Since the data measured by the lidar is averaged every $30 \mathrm{~m}$ in the line-of-sight direction, the result changes according to the position of wake vortex. It involves errors in the wake visualization and the vortex parameter estimation. Therefore, consideration of such error is required for more accurate estimation of the wake vortex parameters based on the lidar measurements.

To evaluate the errors due to the lidar measurement process, the 'pseudo' lidar measurements are applied to artificial wake vortices that are numerically generated by the CFD simulation as shown in Fig. $6^{[14]}$. The pseudo lidar measurement imitates the same situations of scanning and averaging processes as the actual ENRI's lidar described in Sect. II.A. The resulting data is called as 'processed CFD results' in contrast to the original (straight) CFD results. Then, the vortex parameters obtained from the straight and processed CFD results are compared as described in Sect. II.B

The CFD simulation for the artificial wake vortices adopts the three-dimensional compressible Navier-Stokes equations with gravity term assuming an altitude of $170 \mathrm{~m}$. It starts from the initial state including the Hallock-Burham's vortices of which circulation strength corresponds to actual take-off weight of the Boeing 767-300.

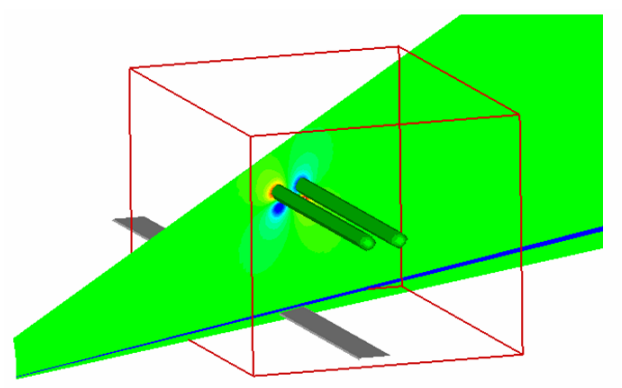

Fig. 6 Schematic of pseudo lidar measurements

\section{A Correction factor}

The difference between the result of the straight CFD and processed CFD are considered as the difference between the actual wake vortices and the actual lidar measurements. Therefore, the ratio between the result of the straight CFD and processed CFD are defined as the "correction factor". Correction factor is used to improve accuracy of the actual lidar measurement of wake vortices. The correction factor is defined as

$$
c_{f a}(t)=\frac{m_{s t}(t)}{m_{p r}(t)},
$$

where $c_{f a}(t)$ is the correction factor at time $t, m_{s t}(t)$ and $m_{p r}(t)$ are a wake vortex parameter (peak velocity, core radius and circulation) of the straight and processed CFD results, respectively. Then, the correction factor is applied to the velocity and core radius of the actual lidar measurements, respectively. Furthermore, circulation is obtained from Eq. (1) with corrected velocity and radius. Thus, the correction factor of circulation is not utilized directly.

\section{Results}

\section{A. Influence of surrounding wind conditions}

To investigate the influence of the surrounding wind conditions on the convection of 
wake vortices, the time history of the wake vortex position should be classified based on the surrounding wind velocities and directions as shown in Fig. 7. The horizontal axis in the figure represents the time from the start of the measurement, and the vertical axis represents the distance from the center of the runway to the center of the wake vortex. The gray area (105 $\mathrm{m}$ width) shows the runway (interval of white broken line: $45 \mathrm{~m}$ ) and the resolution power $(30 \mathrm{~m})$ added to both ends of the runway. A total of 162 cases are classified to three patterns of surrounding wind velocity; 0 to $4 \mathrm{kt}, 4$ to $8 \mathrm{kt}$ and 8 to $12 \mathrm{kt}$, and four patterns of surrounding wind directions; $0 \mathrm{deg}$ (head wind), $45 \mathrm{deg}, 90 \mathrm{deg}$ (crosswind) and undefined wind due to low surrounding wind velocity (this case is referred as variable (VRB)). Bars show the core diameters of vortices.

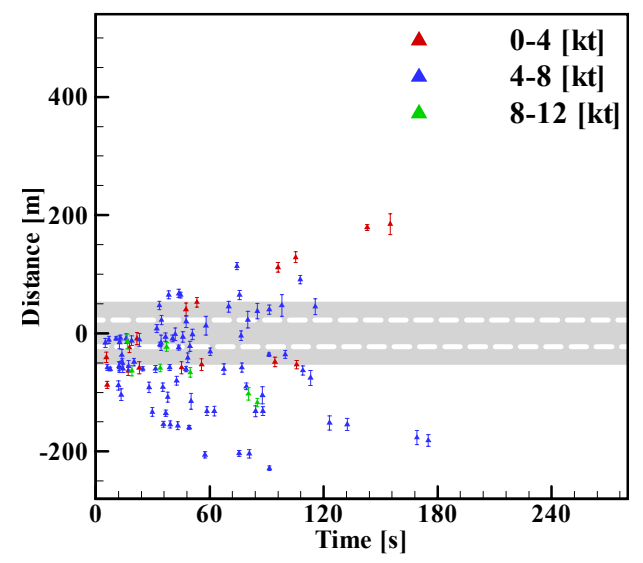

(a)

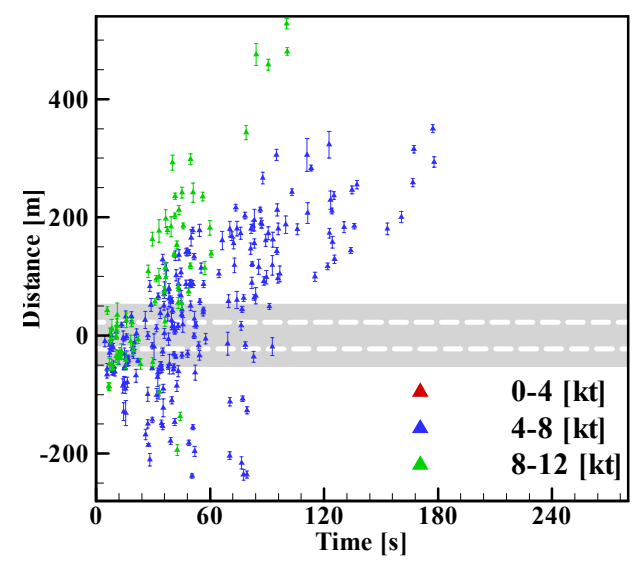

(c)

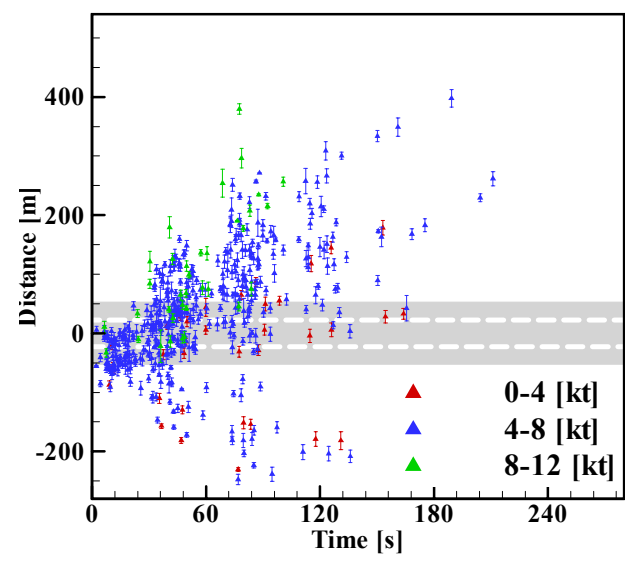

(b)

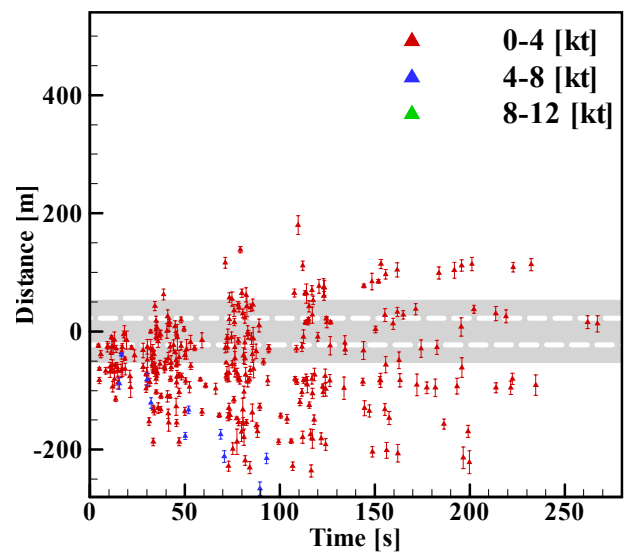

(d)

Fig. 7 Time histories of vortex position for various wind conditions. The surrounding wind directions are classified into (a): $0 \mathrm{deg}$, (b): $45 \mathrm{deg}$, (c): $90 \mathrm{deg}$ and (d): VRB, where the surrounding wind velocity is indicated by red: 0 to $4 \mathrm{~m} / \mathrm{s}$, blue: 4 to $8 \mathrm{~m} / \mathrm{s}$ and green: 8 to $12 \mathrm{~m} / \mathrm{s}$, respectively

From the comparison between the crosswind cases shown in Fig. 7 (c) and Fig. 7 (d), the wake vortices in the crosswind cases disappear more quickly from the runway than in the VRB cases. In addition, the wake vortices in the cases of strong crosswind move faster than in the cases of weak crosswind. This trend can also be seen in the case of diagonal wind as shown in Fig. 7 (b). In the VRB cases in Fig. 7 (d), the vortices reside on the runway for $180 \mathrm{~s}$. This time corresponds to the separation minimum which is conventionally set for a medium or light aircraft following a heavy aircraft. Therefore, if the circulation of residing wake vortex is strong, the conventional separation minimum may not be long enough to avoid the wake turbulence when the surrounding wind is very weak. 
The residence time of wake vortex is investigated for each surrounding wind condition. This time is defined as the period until the center of the wake vortex disappears from the gray area in Fig. 7. Figure 8 shows the average residence time of vortices in each surrounding wind direction and velocity case. The average residence time exceeds two minutes at $4 \mathrm{kt}$ or smaller surrounding wind velocity regardless of the surrounding wind direction. The time of two minutes corresponds to the separation minima when both the leading and following aircrafts are heavy ones. The wake vortices reside on the runway for a long time when the surrounding wind velocity is small regardless of the surrounding wind direction. When the crosswind (including the diagonal direction) has a velocity of $4 \mathrm{kt}$ or above, the wake vortices disappear from the runway in about one minute. Therefore, these results imply a need to study the separation minima when a surrounding wind is weak. On the other hand, the possibility of a shorter separation minimum can be seen in the cases of strong crosswind.

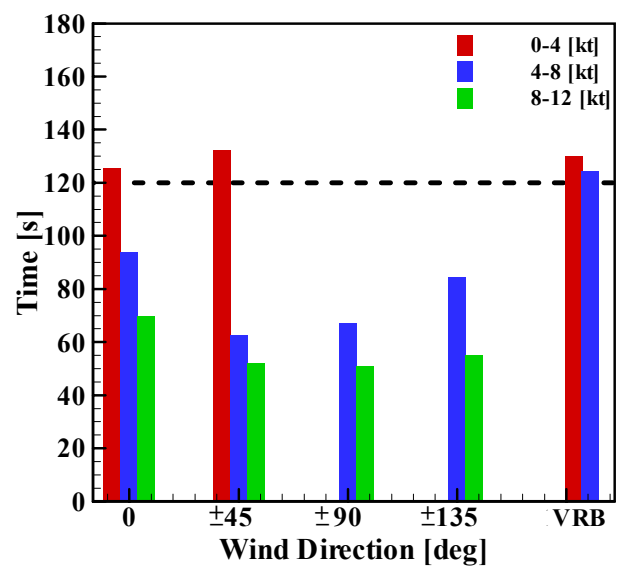

Fig. 8 Average residence time of vortices in each surrounding wind direction and velocity case.

\section{B. Estimated errors due to lidar measurements}

The vortex parameters obtained from the straight CFD results (original calculated results) and processed CFD results are compared as shown in Fig. 9. The vertical axis represents the peak velocity in Fig. 9 (a), the core radius in Fig. 9 (b), the circulation in Fig. 9 (c). The horizontal axis in the figure represent the time from the initial state. The artificial wake vortices generated by the CFD simulation move down towards the ground in about $100 \mathrm{~s}$ after the initial state. When the artificial wake vortices reach the ground, the accurate evaluation becomes difficult because a sudden decay of the artificial wake vortices occurs in the CFD simulation. To easily compare the time variation of vortex parameters, fitting curves of vortex parameters during the initial $100 \mathrm{~s}$ are considered. The solid and broken lines of the straight CFD results are drawn based on the single case, because there is little influence in the vortex parameters due to the accompanying vortex in the CFD simulation. The fitting curve for the processed CFD results is drawn by averaging the 12 cases with different center positions of vortices from the runway center $(0,10,20$ and $30 \mathrm{~m})$ and intervals between left and right vortices $(10,20$ and $30 \mathrm{~m})$ up to $100 \mathrm{~s}$. The fitting curves after $100 \mathrm{~s}$ are simply extrapolated. "Error" in this figure indicates an estimated error in the lidar measurements. The estimated error was obtained from the maximum difference between the each averaged value and each value of 12 cases in the vortex parameters up to $100 \mathrm{~s}$. 


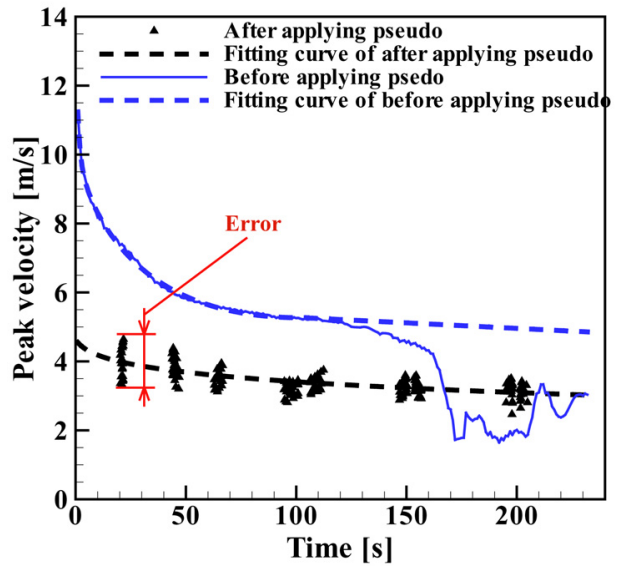

(a)

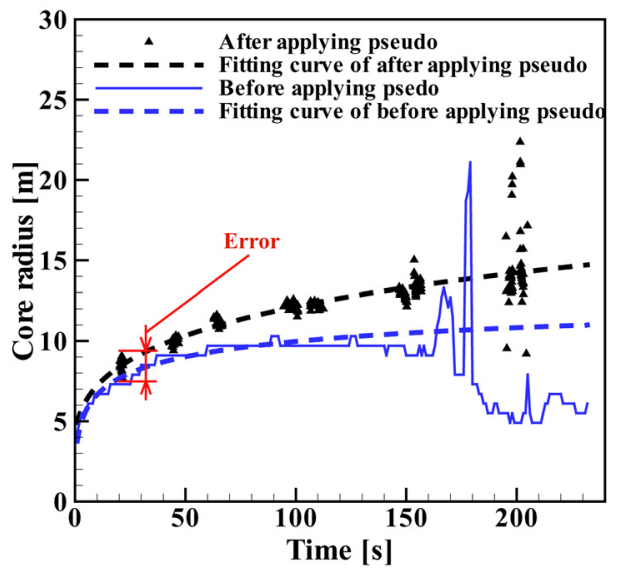

(b)

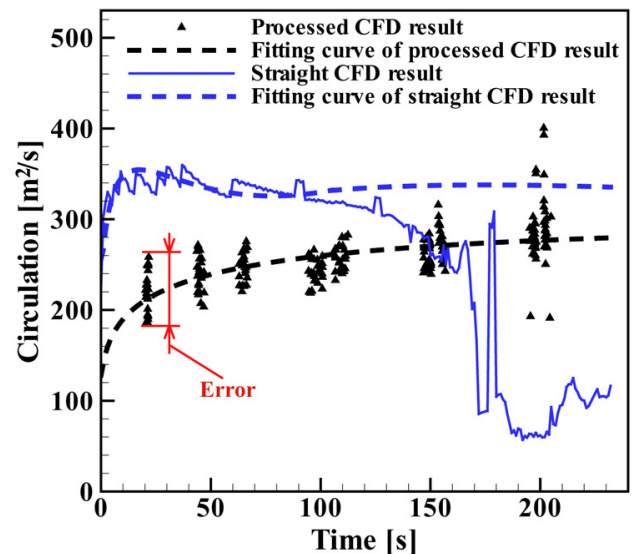

(c)

Fig. 9 The comparison of vortex parameters obtained from straight and processed CFD results, in which (a) is peak velocity, (b) is core radius, and (c) is circulation (solid line: straight CFD results, broken line: fitting curve for the straight CFD results, triangles: processed CFD results, dashed line: fitting curve for processed CFD results).

From Fig. 9 (a), the peak velocities in both straight and processed CFD results decrease with time, and the difference between the peak velocity of these cases also decrease. A sharper velocity peak leads to be blunted more greatly through the lidar measurement process. In Fig. 9 (b), on the other hand, the difference between the core radius of the straight and processed CFD results increases with time. The core radius using the lidar measurement grows larger with time. The circulation is determined by the core radius as shown in Eq. (1). Thus, the accuracy of circulation calculation strongly depends on the core 
radius. Then, based on the results shown in Fig. 9, correction factors are obtained from Eq. (3) as shown in Fig. 10.

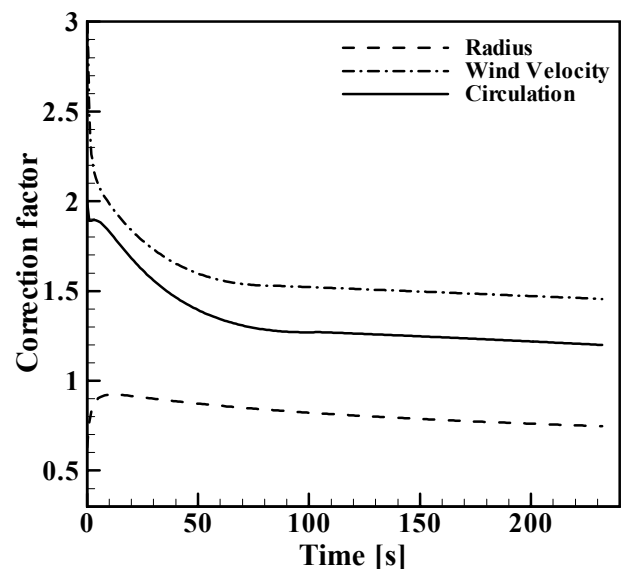

Fig. 10 Correction factors (broken line: core radius, dashed line: peak velocity, solid line: circulation)

\section{Corrected actual lidar measurements}

The actual lidar measurements were corrected using the correction factors shown in Fig. 11. The vertical axis of this figure represents the velocity of the wake vortex, and the horizontal axis represents the core radius. This figure shows that the velocity of the wake vortex becomes larger by using the correction factors, and the core radius becomes slightly small.

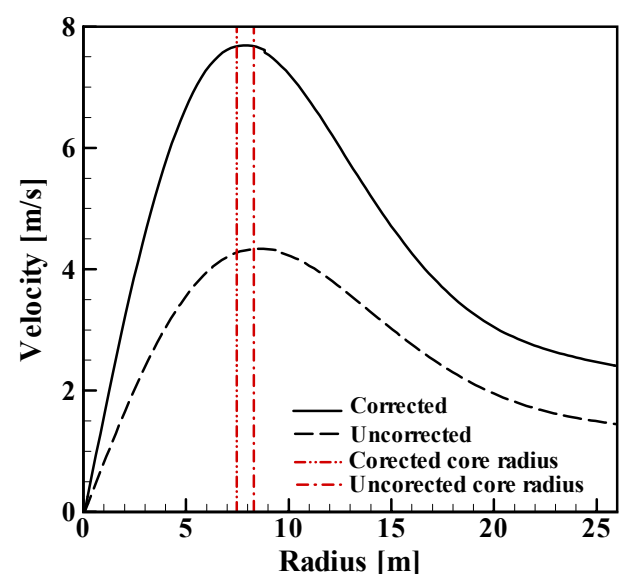

Fig. 11 Example of corrected results at $20 \mathrm{~s}$ from initial state (broken line: uncorrected velocity, solid line: corrected velocity, doubly dashed line: uncorrected core radius, dashed line: corrected core radius)

Both the uncorrected and corrected results for 24 case of the actual lidar measurement are shown in Figs. 12 to 14. These cases are selected because of their weak surrounding wind velocity ( 0 to $4 \mathrm{kt}$ ), since the CFD simulation in Ref. 14 does not consider ambient winds. The solid lines in Figs. 12 to 14 are fitting curves for the lidar measurements, and the broken line in Fig. 12 is the theoretical distribution of the core radius against time ${ }^{[13]}$, and the broken line in Fig. 13 is the decay process model of circulation for the wake vortex given in Ref. 15. The decay process model was based on the data from measurements using a continuous wave (cw) lidar with high resolution power for about 140 cases. In those measurements, an atmospheric state was peaceful and wake vortices were considerably far 
from the ground. Thus, the data measured by the cw lidar does not include ground effect. Furthermore, the broken line in Fig. 14 represents the peak velocity of the decay processes model obtained by applying the circulation decay process model and the theoretical distribution of the core radius against time to Eq. (1). Error bars in Figs. 12 to 14 were obtained in Fig. 9.

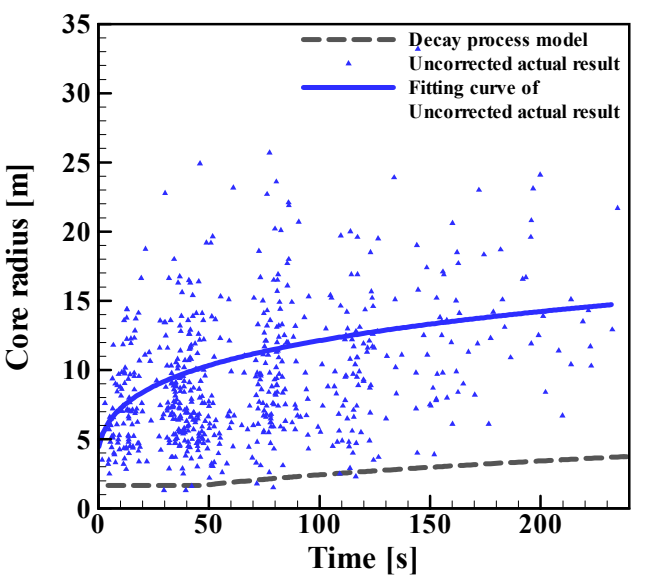

(a)

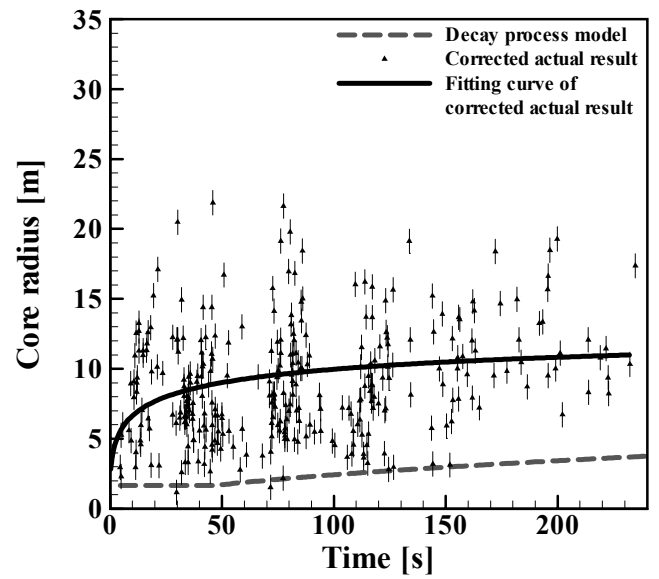

(b)

Fig. 12 Comparisons of the core radius ((a) uncorrected and (b) corrected results)

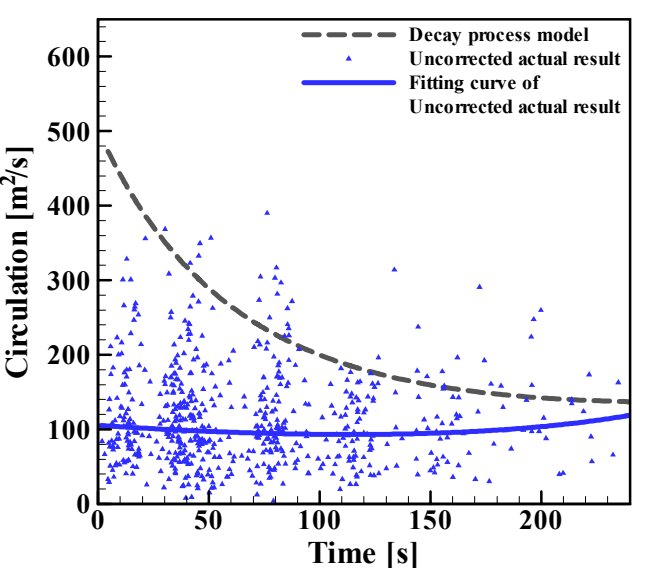

(a)

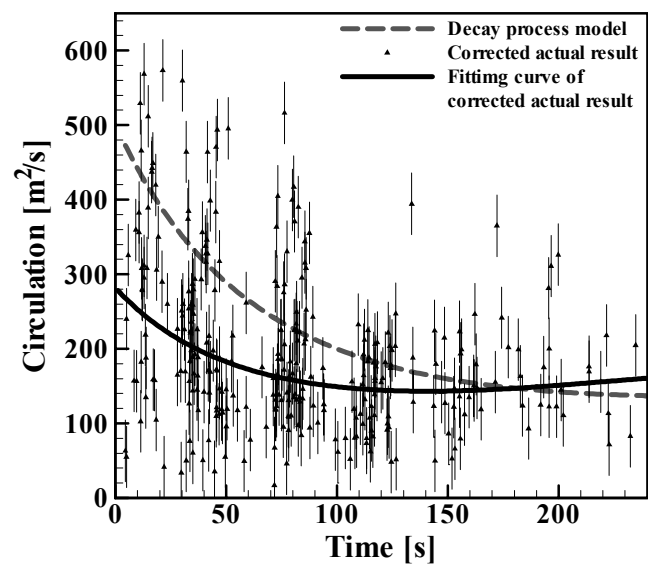

(b)

Fig. 13 Comparisons of the circulation ((a) uncorrected and (b) corrected results)

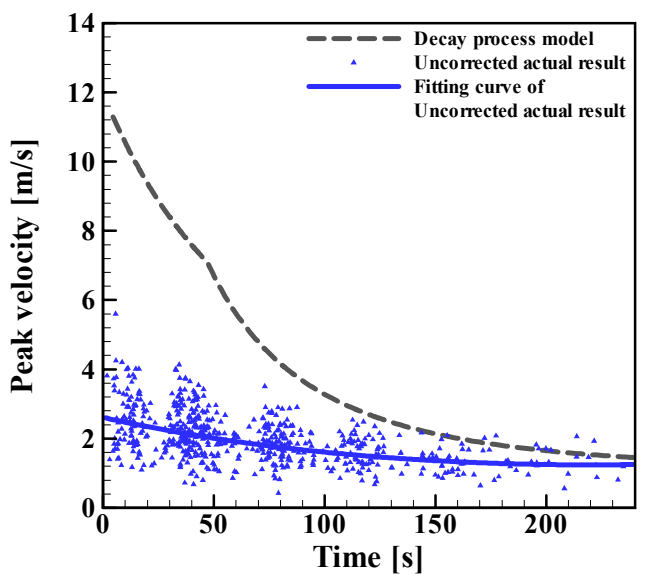

(a)

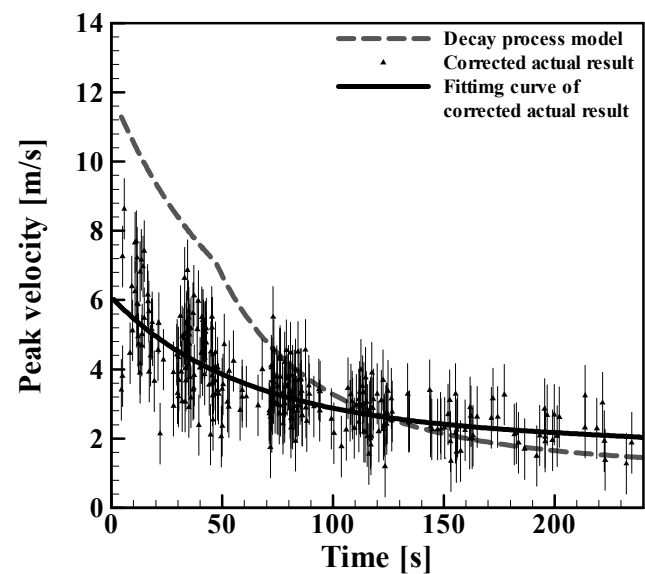

(b)

Fig. 14 Comparisons of the peak velocity ((a) uncorrected and (b) corrected results) 
In Fig. 12, although it is difficult to find the distribution of the core radius from the measured plots themselves, the fitting curve in Fig. 12 (b) appears more clearly compared to the uncorrected results in Fig. 12 (a). There is still large discrepancy between the corrected results and the theory.

The uncorrected circulation in Fig. 13 (a) appears almost constant. On the other hand, the corrected circulation in Fig. 13 (b) approaches the decay process model, and a slight tendency of attenuation can be seen. The main difference exists in the initial period, but corrected circulation agrees with the decay process model after roughly $120 \mathrm{~s}$ i.e. around the separation minima.

In Fig. 14, the peak velocity of the decay process model is calculated by using the theoretical distribution of the core radius against time. Although the model predicts a rapid reduction of the peak velocity, the corrected peak velocity remains higher for roughly $100 \mathrm{~s}$. The peak velocity obtained from the decay process model may not be correct, since the present theoretical diffusion of the core radius is very simple.

Overall, for the case of weak surrounding winds, the use of the correction factors has an advantage to accurately predict wake vortex parameters around the separation minima. However, neither the decay process model nor the present correction factors consider the weather condition. More researches are needed to identify the behavior of wake vortices.

\section{Conclusion}

The influence of the surrounding wind on the behavior of wake vortices is investigated using lidar measurements on actual aircraft at the Sendai airport. The actual lidar measurements are classified based on the surrounding wind velocity and direction. The wake vortices in the crosswind case disappear more quickly from the runway than those of the VRB and head wind cases. In addition, the wake vortices in the case of large crosswind move faster than in small crosswind.

The correction factor and the estimated error were obtained by comparing processed CFD results with straight CFD results. Furthermore, the actual lidar measurements were corrected by applying the correction factors used for weak surrounding wind. The use of correction factors has an advantage to accurately predict wake vortex parameters around the separation minima.

\section{Acknowledgment}

We would like to appreciate the Sendai airport meteorological observatory for providing us weather data, the Mitsubishi Electric Corporation for assisting our experiments, and Dr. Nobuyuki Kaku from ENRI (retired) for his kind supports.

\section{References}

[1] Speijker, L., Baren, G. V., Sherry, L., Shortle, J. F., “ASSESSMENT OF WAKE VORTEX SEPARATION DISTANCES USING THE WAVIR TOOLSET,” Digital Avionics Systems Conference 2004, pp. 2.E.2-1-11, 24-28, October 2004.

[2] Shortle, J. F., Allocco, M., “APPLYING QUALITATIVE HAZARD ANALYSIS TO SUPPORT QUANTITATIVE SAFETY ANALYSIS FOR PROPOSED REDUCED WAKE SEPARATION CONOPS," 6th USA/Europe ATM R\&D Seminar, Baltimore, MD, Paper 82, April 2005.

[3] Holzäpfel, F., Gerz, T., Frech, M., Taffemer, A., Köpp, F., Smalikho, F., Rahm, S., Hahn, K. U., Schwarz, C., "THE WAKE VORTEX PREDICTION AND MONITORING SYSTEM WSVBS-PART 1: DESIGN,” 1st CEAS European Air and 
Space Conference, 2007, pp. 2283-3390, 2007.

[4] AIM-Japan compilation association, "AIM-J 2007 the first term version," Japan Aircraft Pilot Association, 9-3, 2007.

[5] EUROCONTROL EXPERIMENTAL CENTRE, "ATC-WAKE Final Report on System Requirements," EEC Note No. 16/03, 2003.

[6] Flight Standards Division, Engineering Department, Civil Aviation Bureau, Ministry of Land, Infrastructure and Transport, "Aeronautical Information Publication," Civil Aviation Bureau, Ministry of Land, Infrastructure and Transport, Jul. 2005.

[7] Frech, M., Holzpäfel, F., "Skill of an aircraft wake-vortex transport and decay model using weather prediction and observation," $12^{\text {th }}$ Conference on Aviation, Range \& Aerospace Meteorology paper, January 2006.

[8] Kaku, N., "Observation of Wake Turbulence using Laser," IEICE technical report Vol.106, No. 311(20061019) pp. 13-16, 2006 (in Japanese).

[9] Frehlich, R., "Velocity Error for Coherent Doppler Lidar with Pulse Accumulation," Journal of Atmospheric and Oceanic Technology, Vol. 21, Issue 6, June 2004, pp. 905-920.

[10] Ogasawara, T., Misaka, T., Ogawa, T., Obayashi, S., Yamada, I., "Effect of a Surrounding Atmosphere to Wake Vortex," Proceeding of 45th Aircraft Symposium, pp.1305-1306, 2007 (in Japanese).

[11] Matsuda, T., Ando, T., Furuta, M., Hirano, Y., Nakayama, T., and Komiyama, S., "Upper-air wind observation with an all-fiber Coherent Doppler lidar system (Comparison of ultrasonic wind measurement sensor)," Digest of 24th Laser Sensing Symposium, pp. 1-3, 2005 (in Japanese).

[12] Hinton, D. A., Tatnall, C. R., "A Candidate Wake Vortex Strength Definition for Application to the NASA Aircraft Vortex Spacing System (AVOSS)," NASA-TM 110343, pp. 3-26, 1997.

[13] Gerz, T., Holzäpfel, F., Darracq, D., "Aircraft Wake Vortices -A position paper-," http://www.pa.op.dlr.de/wirbelschleppe/WakeVortex.html, 2001.

[14] Misaka, T., Ogasawara, T., Obayashi, S., Yamada, I., Okuno, Y., "Investigation of Lidar Measurement Integration Method for Wake Turbulences Simulation," Proceeding of 45th Aircraft Symposium, pp.1309-1312, 2007 (in Japanese).

[15] Holzäpfel, F., "Probabilistic Two-Phase Wake Vortex Decay and Transport Model," Journal of Aircraft, Vol.40, No. 2, pp. 323-331, 2003. 